\title{
TEST MODULES AND COGENERATORS
}

\author{
PETER VÁMOS
}

\begin{abstract}
If $\operatorname{Hom}_{R}(A, T)=0$ implies that $A=0$ for all $R$-modules $A$, then the $R$-module $T$ is a test module. The ring $R$ is said to be a TC-ring if every test module is a cogenerator. If $S$ is a simple module over a $T C$-ring then $\operatorname{End}_{R} E(S)$ is a local semifir. A commutative ring $R$ is a $T C$-ring if and only if $R_{M}$ is a P.I.D. for all maximal ideals $M$ of $R$.
\end{abstract}

All rings have an identity element and modules are unitary left modules. For an $R$-module $A, E(A)$ denotes the injective envelope of $A$ and $A^{k}$ the $k$ fold direct sum of $A$. Let $R$ be a ring and $T$ an $R$-module. We say that $T$ is a test module if $\operatorname{Hom}_{R}(A, T)=0$ implies $A=0$ for all $R$-modules $A$. The following result was proved by $\mathrm{T}$. Cheatham and R. Cumbie.

Theorem A [1, Theorem 3]. For a ring $R$ the following are equivalent:

(i) every test module is a cogenerator;

(ii) for all simple modules $S, E(S)$ is contained in every nonzero factor of $E(S)$.

A ring satisfying conditions (i) and (ii) in Theorem A is called a TC-ring.

Let $R$ be a $T C$-ring, $S$ a simple $R$-module and set $E=E(S)$. Some of the properties of $E$ which follow easily from Theorem $\mathrm{A}$ are set out in the following two lemmas.

LeMma 1. Let $A$ be a nonzero factor module of $E$. Then the following assertions are true:

(i) the socle of $A$ is a direct sum of copies of $S$ and $A$ is an essential extension of its socle;

(ii) if the socle of $A$ is isomorphic to $S^{k}$ for some integer $k>0$, then $A \approx E^{k}$;

(iii) if $f \in \operatorname{End}_{R} E$ and $f \neq 0$ then $f$ is onto;

(iv) if $S^{\prime}$ is another simple $R$-module such that $S \approx S^{\prime}$, then $\operatorname{Hom}_{R}\left(E, E\left(S^{\prime}\right)\right)$ $=0$.

Proof. Property (i) was noticed in [1] and is a simple consequence of condition (ii) in Theorem A. Now assume that the socle of $A$ is $S^{k}$ for some $k>0$. Since $A$ contains $E$ we have $A=E \oplus A^{\prime}$ and $A^{\prime} \neq 0$ if $k>1$. Since $A^{\prime}$ is again a factor of $E$ it has, in turn, a summand isomorphic to $E$ if $k>1$. Continuing this we obtain $A \approx E^{k}$. Next, let $f \in \operatorname{End}_{R} E, f \neq 0$. Then $f(E) \subseteq E$ and $f(E)$ contains a copy of $E$. Since $E$ is indecomposable, $f(E)=E$. Finally (iv) is immediate from (i).

Received by the editors November 10, 1974 and, in revised form, September 11, 1975.

AMS (MOS) subject classifications (1970). Primary 13C10; Secondary 16A06.

Key words and phrases. Test module, cogenerator, indecomposable injective, endomorphism ring, semifir, P.I.D. 
We remark that in view of (iv) in Lemma 1 the ' cofinitely' generated modules over a $T C$-ring split into their ' homogeneous' components. For the details the reader is referred to [2, Theorem 4.26].

LemMa 2. Let $Q=\operatorname{End}_{R} E, f_{1}, \ldots, f_{k} \in Q$ and assume that the intersection $\operatorname{Ker} f_{1} \cap \cdots \cap \operatorname{Ker} f_{k}$ is irredundant. Then the natural monomorphism

$$
h: E /\left(\operatorname{Ker} f_{1} \cap \cdots \cap \operatorname{Ker} f_{k}\right) \rightarrow E / \operatorname{Ker} f_{1} \oplus \cdots \oplus E / \operatorname{Ker} f_{k}
$$

is an isomorphism. In particular $\operatorname{Ker} f_{i}+\left(\cap_{j \neq i} \operatorname{Ker} f_{j}\right)=E$ for all $1 \leqslant i \leqslant k$.

Proof. It is well known that $h$ is an essential monomorphism (e.g. [2, Theorem 4.9]). Also, $E /\left(\operatorname{Ker} f_{1} \cap \cdots \cap \operatorname{Ker} f_{k}\right)$ is injective by (ii) of Lemma 1. Thus $h$ is an isomorphism. The second part of Lemma 2 now follows from the fact that $h\left(\operatorname{Ker} f_{i}\right)=\bigoplus_{j \neq i} E / \operatorname{Ker} f_{j}$ and $h\left(\cap_{j \neq i} \operatorname{Ker} f_{j}\right)=E / \operatorname{Ker} f_{i}$.

By a local ring we mean a ring with a unique maximal (left) ideal.

Proposition 3. The ring of endomorphisms $Q$ of $E$ is a local semifir i.e. $Q$ is a local domain and every finitely generated (left) ideal of $Q$ is free.

Proof. Since $E$ is indecomposable, $Q$ is local and (iii) of Lemma 1 implies that $Q$ is a domain. Let $L=Q f_{1}+\cdots+Q f_{n}, f_{1}, \ldots, f_{n} \in Q$ be a left ideal of $Q$. We may assume that $L \neq 0$ and that $\operatorname{Ker} f_{1} \cap \cdots \cap \operatorname{Ker} f_{n}=\operatorname{Ker} f_{1}$ $\cap \cdots \cap \operatorname{Ker} f_{k}$ for some $1 \leqslant k \leqslant n$ with $\operatorname{Ker} f_{1} \cap \cdots \cap \operatorname{Ker} f_{k}$ irredundant. If $f \in L$ then $\operatorname{Ker} f \supseteq \operatorname{Ker} f_{1} \cap \cdots \cap \operatorname{Ker} f_{k}$ and a standard argument shows the existence of $g_{1}, \ldots, g_{k} \in Q$ such that $f=g_{1} f_{1}+\cdots+g_{k} f_{k}$. (See e.g. [2, Theorem 5.15, p. 144].) Hence $L=Q f_{1}+\cdots+Q f_{k}$. We claim $L$ is free on $f_{1}, \ldots, f_{k}$. Let $g_{1} f_{1}+\cdots+g_{k} f_{k}=0, g_{1}, \ldots, g_{k} \in Q$ and suppose that $g_{i}(a) \neq 0$ for some $a \in E$ and $1 \leqslant i \leqslant k$. Put $A=\cap_{j \neq i} \operatorname{Ker} f_{j}$. Then $E=\operatorname{Ker} f_{i}+A$ by Lemma 2 and $f_{i}(A)=E$. Now $a=f_{i}(b)$ for some $b \in A$ and $0=\left(g_{1} f_{1}+\cdots+g_{k} f_{k}\right)(b)=g_{i} f_{i}(b)=g_{i}(a) \neq 0$. Thus $g_{i}=0$ for all $1 \leqslant i \leqslant k$ and $L$ is free.

THEOREM 4. For a commutative ring $R$ the following are equivalent:

(i) $R$ is a TC-ring;

(ii) $R_{M}$ is a P.I.D. for all maximal ideals $M$ of $R$;

(iii) every nonzero factor module of $E(R / M)$ is isomorphic to $E(R / M)$ for all maximal ideals $M$ of $R$.

Proof. The implication (iii) $\Rightarrow$ (i) is clear and the equivalence of conditions (ii) and (iii) is a special case of the Corollary in [3].

Assume (i) and let $M$ be a maximal ideal of $R$. Set $E=E(R / M)$. Then $E$ is, in a natural way, an $R_{M}$ module and it is the $R_{M}$-injective envelope of the only simple $R_{M}$ module $R / M \approx R_{M} / R_{M} M$. Then $R_{M}$ is also a $T C$-ring by (ii) of Theorem A. In other words we may assume that $R$ is a local ring with maximal ideal $M$. Then $E$ is a cogenerator and we may assume that $R$ is a subring of $\operatorname{End}_{R} E$. Hence $R$ is a domain by Proposition 3. Let $f, g \in R$. We want to show that either $R f \supseteq R g$ or $R g \supseteq R f$. We may assume that $f g \neq 0$. If $\operatorname{Ker} f \cap$ $\operatorname{Ker} g$ were irredundant then $E=\operatorname{Ker} f+\operatorname{Ker} g$ by Lemma 2. But Ker $f g \supseteq \operatorname{Ker} f+$ $\operatorname{Ker} g$ and $f g \neq 0$. Therefore either Ker $f \supseteq \operatorname{Ker} g$ or $\operatorname{Ker} g \supseteq \operatorname{Ker} f$. Next, the fact that $E$ is the minimal injective cogenerator implies that $\operatorname{Ann}_{R} \operatorname{Ann}_{E} L=L$ for all 
ideals of $L$ of $R$ (see [2, p. 148, (5.4.3)]). This, in turn, yields $R f \supseteq R g$ or $R g \supseteq R f$. Thus $R$ is a valuation domain. Let $L$ be a nonzero ideal of $R$ and $a \in L, a \neq 0$. Set $K=M a$. Since the ideals of $R$ are totally ordered we have $K \subset L, K$ is irreducible and $R / K$ is an essential extension of $R a / K \approx R / M$. Therefore $\bar{E}(R / K) \approx E$ and the natural epimorphism $R / K \rightarrow R / L$ extends to a homomorphism $E \rightarrow E(R / L)$. This shows that $R / L$ is contained in a nonzero factor of $E$ and the socle of $R / L$ is nonzero by Lemma 1 . Since $L$ is irreducible, we obtain that $E(R / L)$ $\approx E$. Accordingly, $R / L$ is Artinian for all nonzero ideals of $R$ by [2, Theorem 3.21]. But this can only happen if $R$ is a field or a rank-one discrete valuation ring. In either case, $R$ is a P.I.D.

\section{REFERENCES}

1. T. Cheatham and R. Cumbie, Test modules, Proc. Amer. Math. Soc. 49 (1975), 311-314.

2. D. W. Sharpe and P. Vámos, Injective modules, Cambridge Univ. Press, New York, 1972.

3. P. Vámos, A note on the quotients of indecomposable injective modules, Canad. Math. Bull. 12 (1969), 661-665. MR 41 \#190.

Department of Pure Mathematics, The University of Sheffield, Sheffield 10, England 